\title{
A new candidate region for the positional cloning of the $X L P$ gene
}

\author{
A lessandra Bolino ${ }^{1}$, L uo Y in $^{2}$, M arco Seri $^{1}$, R oberto Cusano ${ }^{1}$, R oberta Cinti ${ }^{1}$, \\ A lison Coffey ${ }^{3}$, R obert Brooksbank ${ }^{3}$, Gareth Howell ${ }^{3}$, David Bentley ${ }^{3}$, Jack R Davis ${ }^{4}$, \\ A rpad Lanyi ${ }^{4}$, D oli H uang ${ }^{4}, \mathrm{M}$ arkus Stark ${ }^{2}$, M artina Creaven ${ }^{2}$, L ise Bjørkhaug ${ }^{2}$, \\ Fabrice H eitzmann², J érôme L amartine ${ }^{2}$, Simona G audi², Bakary S Sylla², \\ Gilbert M Lenoir ${ }^{5}$, Elio Castagnola ${ }^{6}$, R affaella Giacchino ${ }^{6}$, G iovanni Porta ${ }^{7,8}$, \\ B runella Franco ${ }^{9}$, M assimo Zollo ${ }^{9}$, Janos Sumegi ${ }^{4}$ and G iovanni R omeo ${ }^{1,2}$ \\ ${ }^{I}$ International X L P Consortium, L aboratorio di G enetica M olecolare, Istituto G aslini, G enova, Q uarto, I taly \\ ${ }^{2}$ International X L P Consortium, G enetic Cancer Susceptibility U nit, International A gency for R esearch on Cancer, \\ Lyon, France \\ ${ }^{3}$ International X L P Consortium, The Sanger Centre, Wellcome Trust G enome Campus, H inxton, Cambridge, UK \\ ${ }^{4}$ International X L P Consortium, D epartment of Pathology and M icrobiology, U niversity of N ebraska M edical \\ Center, O maha, USA \\ ${ }^{5}$ International X L P Consortium, U nit of Viral and Hereditary Factors in Carcinogenesis, International A gency for \\ Research on Cancer, Lyon, France \\ ${ }^{6} \mathrm{D}$ ivisione di, M alattie I nfettive, Istituto G aslini, G enova, Q uarto, I taly \\ ${ }^{7}$ International X L P Consortium, D ipartimento di Patologia U mana ed E reditaria, II Facoltà di M edicina, U niversità \\ di Pavia, I taly \\ ${ }^{8}$ International X L P Consortium, L aboratorio di G enetica U mana, Istituto di Scienze Biomediche S Paolo, Università \\ degli Studi di Milano, I taly \\ ${ }^{9}$ International X L P C onsortium, Telethon Institute of G enetics and M edicine, M ilano, I taly
}

\begin{abstract}
X-linked lymphoproliferative disease (XLP) is an inherited immunodeficiency characterised by selective susceptibility to Epstein-Barr virus and frequent association with malignant lymphomas chiefly located in the ileocecal region, liver, kidney and CNS. Taking advantage of a large bacterial clone contig, we obtained a genomic sequence of 197620 bp encompassing a deletion (XLP-D) of $116 \mathrm{~kb}$ in an XLP family, whose breakpoints were identified. The study of potential exons from this region in 40 unrelated XLP patients did not reveal any mutation. To define the critical region for XLP and investigate the role of the XLP-D deletion, detailed haplotypes in a region of approximately $20 \mathrm{cM}$ were reconstructed in a total of 87 individuals from 7 families with recurrence of XLP. Two recombination events in a North American family and a new microdeletion (XLP-G) in an Italian family indicate that the XLP gene maps in the interval between DXS1001 and DXS8057, approximately $800 \mathrm{~kb}$ centromeric to the previously reported familial microdeletion XLP-D.
\end{abstract}

Keywords: immunodeficiency; intestinal lymphomas; Epstein-Barr virus; familial microdeletions

Correspondence: G Romeo, IA R C, 150 Cours A Ibert Thomas, 69008 Lyon. e-mail: comeo@iarc.fr.

Received 12 M ay 1998; accepted 25 June 1998

\section{Introduction}

$X$-linked lymphoproliferative disease (XLP, originally called D uncan disease; M IM 308240) is a rare, inherited 
immunodeficiency characterised by selective susceptibility to E pstein-Barr Virus (EBV). Whilst more than $98 \%$ of individuals from the general population experience an EBV infection with little or no clinical manifestation, affected individuals, when exposed to EBV infection, develop fatal or chronic infectious mononucleosis, acquired agammaglobulinaemia (or hypogammaglobulinaemia), aplastic anaemia and malignant lymphoma. ${ }^{1}$

The XLP gene was initially mapped by linkage analysis in the $X q 24-q 25$ region at $4 \%$ recombination from the DXS42 locus. ${ }^{2}$ F urther linkage studies established that the $X L P$ gene is telomeric with respect to markers DXS42 and DXS37, at about 1-2\% recombination from $D X S 42(Z=17.5)$ and at $3 \%(Z=11.8)$ recombination from the $D X S 37$ locus. $A$ third marker, DXS12, was found to be closely linked to the disease locus and no recombination $(Z=7.5)$ was demonstrated. ${ }^{3,4}$ A cytogenetically visible deletion in $X$ q25 was observed in an XLP family by Wyandt et al. ${ }^{5}$ Subsequently three constitutional deletions were reported in unrelated $X L P$ patients. In the first one the markers DXS6, DXS739 and DXS100 are missing; in the second one the markers DXS739 and DXS100 are deleted; whilst in the third one only the marker DXS739 is missing. ${ }^{6,7}$

Recently a novel constitutional deletion of no more than $120 \mathrm{~kb}$ in an XLP patient (XLP-D), nested within the three cytogenetically visible deletions, was reported by two of our groups. ${ }^{8,9} \mathrm{~A}$ large scale sequence analysis of the bacterial clones from this region carried out in the present work did not lead to the identification of the X L P gene. In order to establish whether this critical region really represented a candidate interval for the $X L P$ gene we performed linkage analysis on seven unrelated pedigrees. To this end highly polymorphic markers spanning the critical region and covering a genetic distance of approximately $20 \mathrm{~cm}$ were used. $\mathrm{H}$ aplotype reconstruction using these markers allowed us to localise the $X L P$ gene in an interval between DXS8081 and DXS8057, about $800 \mathrm{~kb}$ proximal to the previously reported XLP-D deletion. In addition, the genotyping of an I talian family using DXS8057 led to the identification of a novel constitutional deletion ( $X L P-G$ ) present in one affected member as well as in two carrier females. This genetic and physical mapping defines the location of the XLP gene in the interval between DXS1001 and DXS8057, a new candidate region for the positional cloning of the $X L P$ gene.

\section{Materials and Methods}

Patients and Families

Patient XLP-D has been previously described. ${ }^{8,9}$ Genomic DNA extracted from paraffinated blocks of different tissues of his maternal uncle, who died of fatal infectious mononucleosis, was used for the amplification of EST 74809, xSTS916.283N, DF 83, xSTS46709N, DX S7365, DX S7364, 4670.29 (see results).

In each of the seven families genotyped in the present work (three italian, three N orth-A merican and one Swiss), at least two maternally related male members had features of XLP (life threatening infectious mononucleosis, malignant lymphoma and acquired hypogammaglobulinaemia).

The $K 001$ family (Figure 1 ) is the original D uncan pedigree from D avid Purtilo's X LP R egistry (O maha, NE, USA ). In this six-generation pedigree at least 19 males died of $X$ LP. 1,10

The patient from the GL 01 family (Figure 2) was admitted to the Gaslini Institute (Genova) at the age of 6 years for fever, hepatosplenomegaly and persistent high levels of transaminase enzymes after an EBV infection. The liver and bone marrow biopsies suggested the presence of a lymphoproliferative disease. The proband's mother had a first cousin who died of a lymphosarcoma. A suppressive steroid therapy supported by infusion of immunoglobulins every week was started soon after the diagnosis and followed up at monthly intervals by infusions of immunoglobulins during the past 4 years. At present the clinical condition of the patient is stable.

\section{Amplification and Sequencing of the Junction Fragment of the XLP-D Deletion}

In order to amplify the junction fragment, the following primers were used as standard PCR: 6846U, CAGCA CACTGATGGGTCTTGACT； 15700L， GGACCAGTTTGATA C CTTG TG GAT. Sequencing of the junction fragment was carried out using the purified PCR product and the same primers (see R esults).

Exon Trapping and Sequencing of the $X L P-D$ region $\mathrm{E}$ ach of the cosmids ${ }^{8}$ spanning the critical region were digested with $\mathrm{Bam} \mathrm{HI-Bgl} \mathrm{II,} \mathrm{cloned} \mathrm{in} \mathrm{the} \mathrm{PSPL} 3$ vector. A fter Cos cells transfection, a mini library of the RT-PCR products was constructed for each cosmid as described by Church et al. ${ }^{11}$

The cosmids covering the X LP-D deletion were subcloned on the basis of a detailed restriction map $^{8}$ (and unpublished data, Y in $L$, 1998) and sequenced using Dye Primer cycle sequencing chemistry with an Applied Biosystems 377A automated DNA sequencer. Plasmid and cosmid end sequencing was performed using $D$ ye Terminator sequencing chemistry with Taq polymerase together with T3/T7/Sp6 primers. ${ }^{12}$ Primer walking using $D$ ye Terminator sequencing chemistry was used to complete the sequence of each subclone in addition to confirming sequence overlaps of adjacent subclones. All sequences thus generated were assembled using A pplied B iosystems programs FA CTU RA version 1.2.0 and AUTOA SSE MBLER version 1.3.0.

The prediction of exons was performed on unmasked sequence using GRAIL 1, 1A, 1.3, $2^{13}$ and the Gene-Finder options FEXH, HEXON, FGENEH ${ }^{14}$ through URL http:/ /dot.imgen.bcm.tmc.edu:9331/gene-finder/gf. O nly loci predicted at least three times by different programs were 

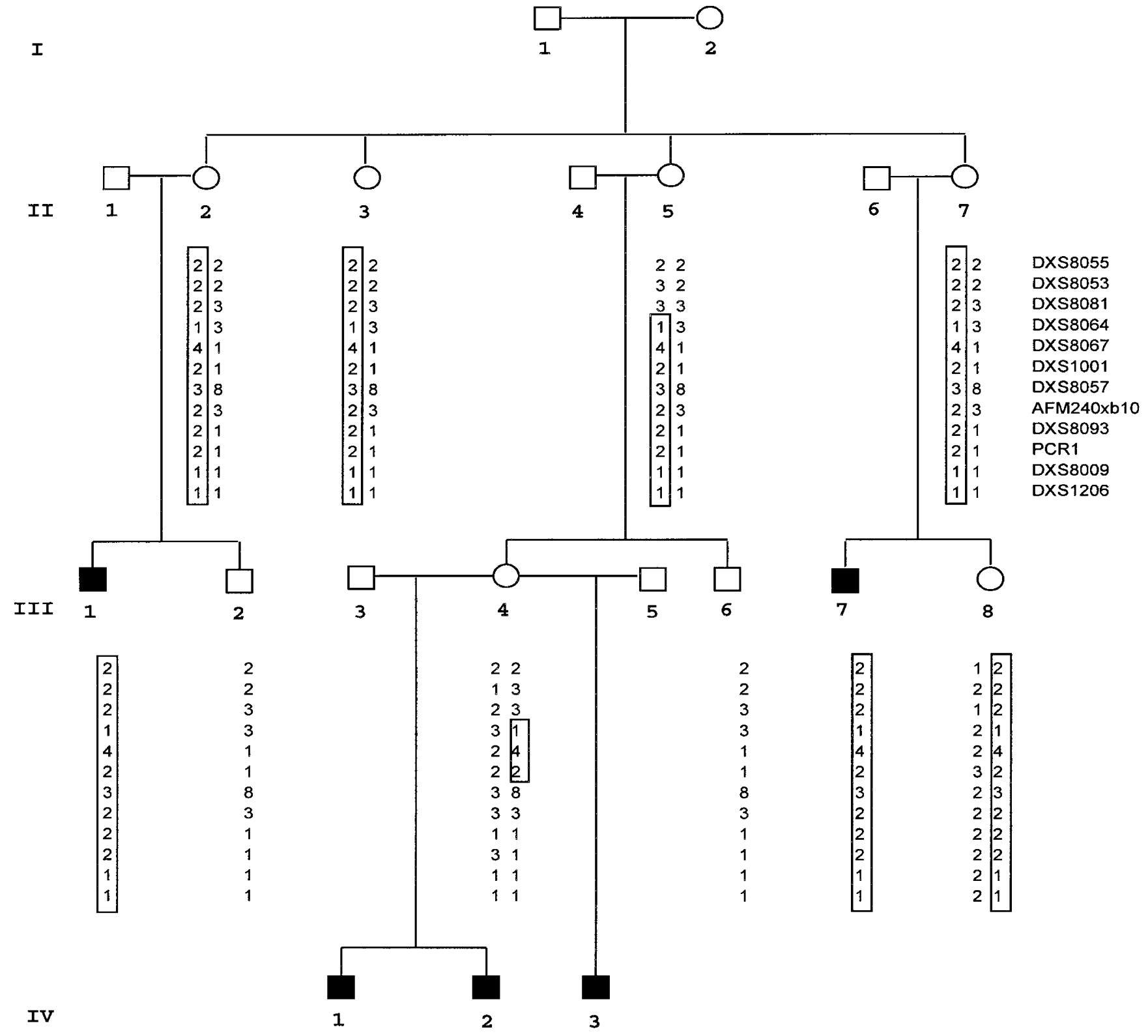

\begin{tabular}{lcc}
2 & 2 & 2 \\
3 & 3 & 3 \\
3 & 3 & 3 \\
\hline 1 & 1 & 1 \\
4 & 4 \\
2 & 4 & 4 \\
\hline 8 & 8 & 8 \\
3 & 3 & 3 \\
1 & 1 & 1 \\
1 & 1 & 1 \\
1 & 1 & 1 \\
1 & 1 & 1
\end{tabular}

Figure $1 \mathrm{H}$ aplotype reconstruction in the K 001 family. O nly markers encompassed by DX S8055 and D X S1206 are shown from centromere (top) to telomere (bottom). The at-risk haplotypes are boxed. The five affected individuals (filled symbols) share alleles only at the D X S8064, D XS8067 and DXS1001 loci. The recombination events demonstrated in this pedigree localise the XLP gene between markers DX S8081 and DXS8057, approximately $800 \mathrm{~kb}$ centromeric to the X LP-D deletion (see text). 
$\mathbf{I}$

II

DXS8055

DXS8067

DXS1001

DXS8057

AFM240xb10

DXS8093

XLP-D-MS

DXS8009

DXS1206

\begin{tabular}{l|l|l}
\hline 1 & \multicolumn{2}{|l|}{2} \\
1 & & 2 \\
1 & 2 & 2 \\
2 & 1 & 3 \\
2 & 1 & 1 \\
2 & 3 & $\Delta$ \\
1 & 3 & 3 \\
1 & 2 & 2 \\
1 & 1 & 2 \\
2 & 2 & 1 \\
1 & 1 & 1
\end{tabular}
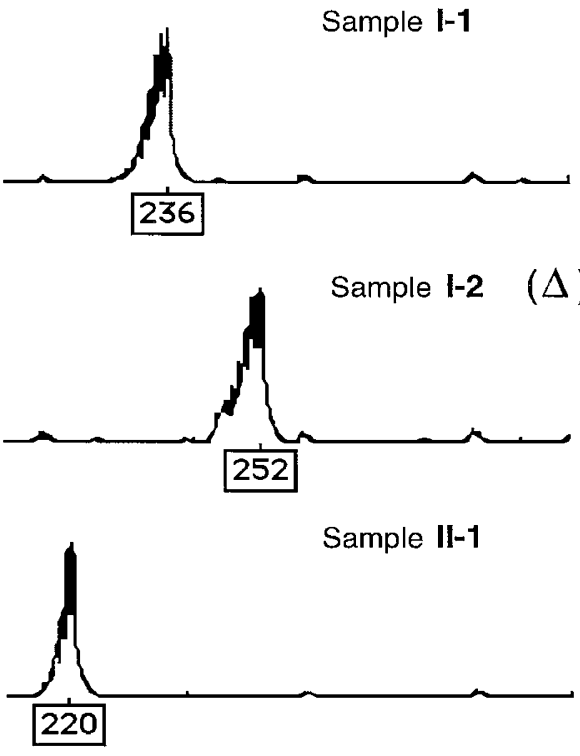

Sample II-2

$(\Delta)$
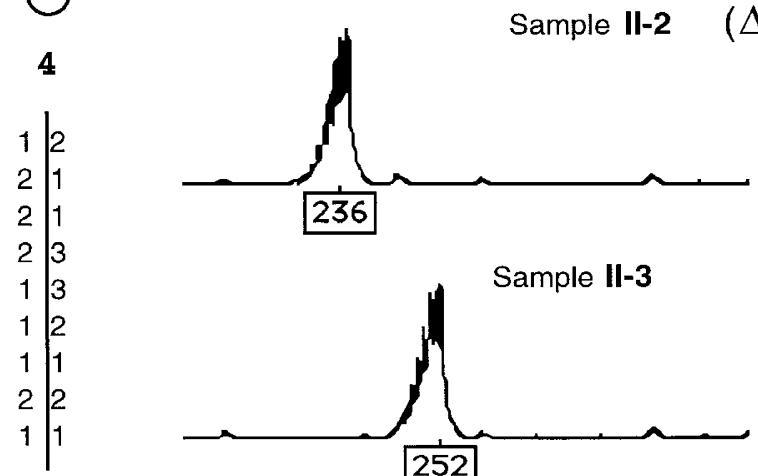

Sample II-3

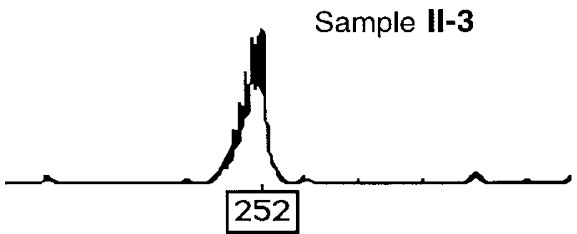

Sample II-4

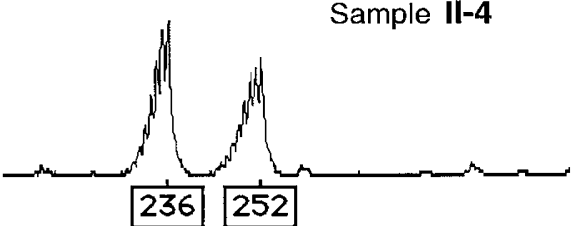

Sample III-1

$(\Delta)$

Sample III-2

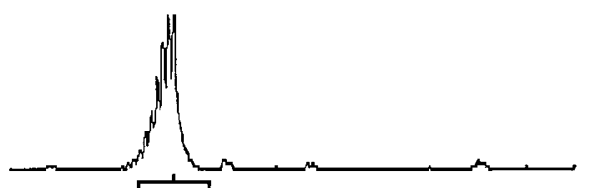

236

Figure 2 Haplotype reconstruction in the G L 01 family. O nly markers encompassed by D X S8055 and D X S1206 are shown. A null allele at the DX S8057 locus (indicated by $\triangle$ ) was demonstrated in III-1, II-2 and was probably also present in I-1. O n the right part of this figure the 3 alleles of DX S8057 are reported in bp: 220 bp corresponds to allele 1, 236 bp to allele 2 and 252 bp to allele 3. 
considered to represent best candidate exons. The entire sequence was also analysed using BLA STN ${ }^{15}$ versus dbEST, dbESTNEW, EMBL, EMNEW, VERTRNA (vertebrate $R N A$ in $E M B L$, non-EST) and $C p G$ island databases, and BLA STX versus SWISSPROT, SPTREMBL and WORM $P E P$ databases. $D$ ata from all the sequence analysis were collected and graphically displayed using an ACEDB database. ${ }^{16}$

\section{Analysis of Mutations and RT-PCR on the four best Candidate Exons}

The primers used to amplify the four best candidate exons ( $A$, $B, C$ and $D$, see results) were designed from the sequence which can be accessed through G enB ank (A F 001905) at the following nucleotide positions: A F 9622-9646, A R 9833-9811; BF 25867-25889, BR 26100-26080; CF 143118-143142, CR 143308-143286; D F 169672-169696, D R 169838-169814. PCR products were analysed on the A BI 377 automatic sequencer. Total RNA was extracted from cell pellet using TRIzol ${ }^{\circledR} R e-$ agent from GIBCOBRL following the protocol recommended by the manufacturer. A pproximately $1 \mu \mathrm{g}$ of total R NA was reverse transcripted using the R NA PCR Core K it from Perkin Elmer. PCR amplification was carried out using the same kit with the addition of Taq Start A ntibody (CLONTECH), mixed 1:1 with the Taq polymerase $5 \mathrm{~min}$ prior to the reaction. The primer sequences (located inside the predicted exons) for potential exon $A, B, C$ and $D$ corresponded to the following nucleotide positions: $A F$ 9702-9725, AR 9769-9746; BF 25965-25988, BR 26032-26009; CF 143174-143195, CR 143233-143212; DF 169730-169755, DR 169801-169776.

\section{Haplotype Reconstruction in Seven Families}

The following microsatellite markers were used to genotype the seven XLP pedigrees: cen - DXS8088 - DXS1220 DXS8055 - DX8053 - DXS8081 - DXS8064- DXS8067 DXS1001 - DXS8057 - AFM 240xb10 - DXS8093 - XLPD-MS - DXS8009 -DXS1206 - DXS8078 - DXS1046 DXS1047 - DXS8041 - tel. The order as well as physical distances for markers from A FM 240xb10 to DX S8078 have been reported in the $4.5 \mathrm{M} \mathrm{b}$ physical contig developed by Porta et al. ${ }^{17}$ The physical order of markers from DX S8088 to D X S8057 and from D X S8078 to D X S8041 was determined on the basis of the PAC contigs available at the Sanger Centre (http://www.sanger.ac.uk/HGP/ChrX/). Finally, the physical distance between the marker D X S8057 and A F M 240xb10 was estimated to be approximately $500 \mathrm{~kb}$ on the basis of the Sanger contig (Coffey A et al, 1998, unpublished data).

A II PCR reactions were performed under standard conditions. Primers used to amplify the marker A FM 240xb10 were reported in the GD B database. X LP-D -M S is a microsatellite marker identified by us from the XLP-D deletion (bp 36843-36882), whose primer sequences and the PCR condition were reported in the GDB database (accession number 6674083). Primers and annealing temperatures used to amplify the remaining microsatellites were reported in the $G$ énéthon genetic map, ${ }^{18}$ except for the following markers, for which primers have been redesigned based on the sequences of these loci reported in the GDB database: DXS8064, forward GCCTGACCAACATGGTGAAC, reverse GGCTGCCA ACTCTTTTCTCC; DXS8093, forward GGCTATTTGCACACCCGGAG, reverse GAGGTGCCGAGAGCAAGAG; DXS8057, forward GCTCTGTA GAAGGGGTAATATGC, reverse CAGCCTCT-
GAAAGTGTTGGG. Genomic DNAs were typed using primers labelled with TET fluorescent amidite and alleles were visualised by an automated sequencer A BI mod 373A and using the $G$ enescan software.

\section{Physical Mapping and FISH}

The PA C clone dJ 1104116 was identified by PCR screening of the PA C library R PCI-5 using primers at the D X S8057 locus. PAC clones dJ 231B20, dJ 1052M 9, dA 1010 7, dA 173I 14, d) $221 \mathrm{H} 19$, d) 369024 , d) 618F 1, d) 693G 4, d) 876G 16 and d) 1104116 were provided by the Sanger Centre. In addition, BAC clones $258 \mathrm{H}$ 9, 99N 24, 63G 16, 281/ 22 were selected by D r. J. Sumegi (1998, unpublished data).

FISH experiments were performed with PAC clones d) 231B 20, dJ 1052M 9, dA 1010 7, dA 173l 14, d) 221H 19, d) 369024 , d) 618F 1, dJ 693G 4, dJ 876G 16 and dJ 1104I 16 on metaphase chromosomes obtained from lymphoblastoid cell line of the G $L 01$ patient, according to a protocol described by Pinkel et $\mathrm{al}^{19}$ with minor modifications.

The following STSs, 281i22-T7, 63g16-Sp6, 258h9-T7, 281i22-Sp6, 63g16-T7, 99n24-T 7 and 258h9-SP6, were obtained through end sequencing of BAC clones 281122, $63 \mathrm{G} \mathrm{16}, 99 \mathrm{~N} 24$, and 258H 9 (Sumegi J et al, 1998, unpublished data). On the other hand, STSs 1052m9-T 7 and 231b20-T7 were constructed with the end sequences of the PA C clones from the Sanger Centre (dJ 1052M 9 and dJ 231B20). A II primer sequences for these STS are available upon request.

\section{Results}

\section{Molecular Characterisation of the XLP-D Deletion}

The total length of the sequencing contig encompassing the XLP-D deletion and consisting of $197620 \mathrm{bp}$ can be accessed (A F 001905). The sequence of the junction fragment was obtained from a purified $0.4 \mathrm{~Kb} P C R$ product (see $M$ aterials and $M$ ethods). A fragment of $116924 \mathrm{bp}$ was missing in patient X L P-D between bases 31089 and 148013 of the wild type sequence. In addition $26 \mathrm{bp}$ between nucleotides 31033 and 31058 were also missing in patient XLP-D, possibly as result of the same event which led to the deletion of $116924 \mathrm{bp}$. Since this $26 \mathrm{bp}$ deletion is not present in $60 \mathrm{X}$ chromosomes from 40 normal control individuals ( 20 males and 20 females), it is unlikely that it represents a polymorphism.

Genomic DNA extracted from paraffin embedded tissues of the maternal uncle of patient XLP-D who died of fatal infectious mononucleosis was found deleted for XLP-D-MS (bp 36843-36882), XSTS916.283N (bp 50458-50618), DF83 (bp 63537-63889), xSTS46709N (bp 109236-109706), DXS7365 (bp 111492-111640), which are deleted in XLP-D, whilst the markers EST 74809 (bp 30111-30460), DXS7364 (bp 156847-156948) and 
4670.29 (bp 174094-174176) are not deleted in either the XLP-D patient or his uncle.

The only significant, potentially coding BLASTN homology found was to $\mathrm{HSXQD},{ }^{20}$ with $100 \%$ identity over more than $1 \mathrm{~kb}$ between $63518-64656$ in XLP-D (78-1216 in HSXQD). However, this sequence was masked by RepeatM asker2 as L 1 element. No significant homologies were found as a result of B LA STX searches. The potential exon analysis of the $197620 \mathrm{bp}$ sequence carried out as described in Materials and $M$ ethods led to the identification of four candidates: A (9702-9769 bp), B (25963-26032 bp) and D (169806-169730 bp) were identified by three independent sequence analysis methods, and potential exon C (143174-143236 bp) was identified twice by these methods and once by exon trapping. Sequencing in $40 \mathrm{XLP}$ patients for these four best candidate exons did not detect any apparent mutation. RT-PCR assay did not identify any significant expression in monocytes, lymphoblastoid cell lines, the neuroblastoma cell line SK-NBE-5Y and the teratocarcinoma cell line BA 129 (data not shown). The screening of human spleen CDNA library with potential exon $C$ as probe did not yield any positive clone.

\section{Haplotype Analysis in the $20 \mathrm{cM}$ Region of Xq25}

The seven available families were typed using the markers reported under $M$ aterials and $M$ ethods which cover a region of approximately $20 \mathrm{cM}$. The results from haplotype reconstruction for five of the seven families analysed are not shown, since no recombination nor microdeletion events were apparent. The seven families show different at-risk haplotypes. Figure 1 reports the haplotype reconstruction in the $\mathrm{K} 001$ family for the regions encompassed by D X S8055 and D XS1206. Since two recombination events occurring through two subsequent generations were found, this pedigree was typed with four additional markers, DXS8088, DXS1220, DXS8053 and DXS8064, in order to define the recombination breakpoints. In the second generation of the $\mathrm{K} 001$ pedigree, three out of four sisters II-2, $\mathrm{II}-3$, and II-7 have the same at-risk haplotype observed in the affected male III-1. The fourth, II-5, shows a first recombination event between markers DXS8081 and DXS8064. In the third generation, the affected males III-1 and III-7 as well as the carrier female III-8 share almost all the at-risk haplotype. A further recombination in the at-risk haplotype was observed between markers DXS1001 and DXS8057 in the III-4 female. Her three sons (IV-1, IV-2, and IV-3) share therefore with these second cousins (III-1 and III-7) alleles only at the DXS8064, DXS8067 and DXS1001 loci. These observations indicate that the $X L P$ gene in this pedigree must be between markers DXS8081 and DXS8057.

\section{Characterisation of a new Microdeletion in an XLP Patient}

Figure 2 shows the results of the genotyping on the GLO1 family. No recombination event was found between the disease gene and any of the markers used. Patient III-1 carried a null allele at the D X S8057 locus in the grand-maternal haplotype, whilst his unaffected brother III-2 inherited the intact grand-paternal haplotype. The same genomic alteration was shown also in their mother II-2, who inherited only the paternal allele 2 , suggesting a genomic deletion of DXS8057. DNA from 40 unrelated XLP patients were amplified using primers at the DXS8057 locus. No additional microdeletion was demonstrated. In addition, genomic D NA from 24 normal males was tested for the same marker. No deletion was found. The absence of deletion in DXS8057 in a total of 64 males indicate that the null allele of this marker is not a deletion polymorphism.

The XLP-G deletion was confirmed and characterised by FISH as well as by STS analysis (Figure 3 ). PA C clones dJ 1104l 16, dJ 876G 16, dJ 693G 4, dJ 231B 20, positive for DXS8057, detected a clear hybridisation signal in lymphoblastoid cell lines from normal controls, but not in patient III-1. The dJ $618 \mathrm{~F} 1$ clone gave a weak hybridisation signal only in $28 \%$ of the metaphases analysed, suggesting that the telomeric breakpoint of the XLP-G deletion is contained in this PAC (Figure 3). Furthermore, PAC clones dJ 36024 , d) $221 \mathrm{H} 19$ and dA $173 \mathrm{l} 14$, telomeric to and partially overlapping with dJ $618 \mathrm{~F} 1$, were all present in the patient III-1. On the other hand, the centromeric clones dA 10107 and dJ 1052M 9 were absent in the XLP-G deletion by FISH analysis suggesting that the centromeric boundary of the deletion locates centromerically to these two PAC clones (Figure 3).

These data were further confirmed by STS analysis. The most centromeric 281i22-T 7 ST S, contained in BA C clone 281I22, is present in patient III-1, whilst STS 1052m9-T7, telomeric to the previous one and contained in clones 281/22, dJ 1052M 9 and dA 10107 , is deleted in patient III-1. The telomeric breakpoint of the deletion is defined by STS 99n24-T7 and marker DXS7144. In particular, STS 99n24-T7 contained in clones 258H 9, 99N 24, dA 173I 14, dJ 221H 19, dJ 369024 , d) 618F 1, dJ 693G 4 and dJ 876G 16 is deleted in patient 

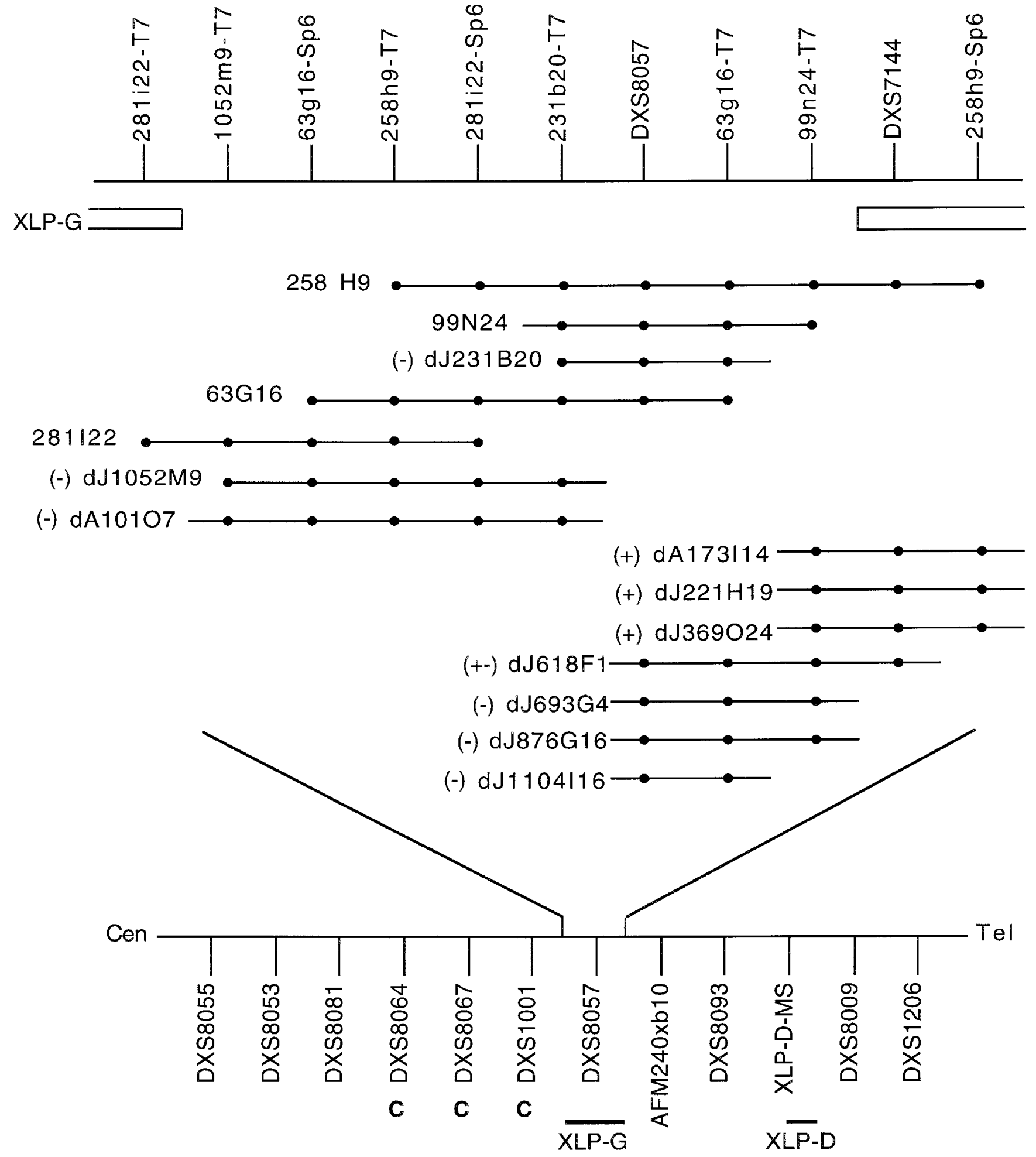

Figure 3 Physical and genetic mapping of the new candidate X L P deletion. The results of FISH analysis are reported on the left of the PAC clones used as probes to analyse metaphases from a lymphoblastoid cell line of patient $\mathrm{G} L 01$. + : clear hybridisation signal, +- : weak hybridisation signal, indicating partial retention of the corresponding genomic region, -: no hybridisation. The centromeric and telomeric breakpoints of the X L P-G deletion indicated in this figure were defined by amplification of the STSs reported at the top (see results). A t the bottom of the figure, the locations of the XLP-G and XLP-D deletions are shown, and the markers concordant in affected individuals of pedigree K 001 are marked C. 
III-1, whilst marker DXS7144 contained in clones $258 \mathrm{H}$ 9, dA $173 \mathrm{I} 14$, dJ $221 \mathrm{H} \mathrm{19}$, dJ 369024 and dJ 618F 1 is present in III-1. In conclusion, the two breakpoints of the X LP-G deletion fall in the centromeric 281I 22 and the telomeric dJ 618F 1 clones, respectively. $0 \mathrm{n}$ the basis of the sizes of the BAC and PAC clones contained in the contig of Figure 3, the X LP-G deletion is estimated to span approximately $250-300 \mathrm{~kb}$ of genomic D NA.

\section{Discussion}

A fter defining the breakpoints of the XLP-D deletion, the entire DNA segment absent in this patient (116924 bp) and the two flanking sequences (31089 bp centromerically and 49606 bp telomerically) were analysed and four potential exons were identified on the basis of their prediction by at least three different prediction methods (see Results). None of them matched the existing ESTs, nor had any significant homology with known proteins. No apparent point mutation was found in genomic DNA from $40 \times \mathrm{XL}$ patients, nor was any significant expression detected for the four putative exons in four different cell lines: monocyte, Iymphoblastoid, neuroblastoma, SK -NBE $5 Y$ and teratocarcinoma BA 129. The possibility of a sporadic association between the XLP clinical phenotype and the deletion occurring in patient XLP-D was therefore taken into account, in spite of the familial recurrence of the XLP-D deletion (see Results). A s alternative hypotheses, we considered that this deletion (a) might contain only a very small portion of the X LP gene; (b) might be located outside the XLP gene, influencing its expression by long-range positional effect. $^{21}$

Following the latter hypothesis we focused our efforts on further genetic mapping of the $X L P$ region. We therefore genotyped seven pedigrees for some of the polymorphic markers available over a wide interval in the $X$ q25 region.

The recombinations found in kindred $\mathrm{K} 001$ define a new location of the XLP gene between centromeric marker DX S8081 and the telomeric marker D X S8057. On the other hand, the X LP-G deletion in family G L 01 which involves only the $D X S 8057$ but not the proximal marker DXS1001 nor the distal marker XLP-D-MS suggests that the $X L P$ gene maps distal to $D X S 1001$ and centromeric to XLP-D deletion. The genetic data obtained from $\mathrm{KO} 01$ and GLO1 pedigrees are thus concordant, pointing to a second candidate region for the XLP gene delimited by DXS1001 and DXS8057
(Figure 3), which spans at least $2 \mathrm{Mb}$. The physical distance between the telomeric boundary of this second candidate region and the centromeric breakpoint of the $X$ LP-D deletion is approximately $800 \mathrm{~kb}$.

The discrepancy arising from the observation of two candidate regions may be explained by the existence of a second non-apparent mutation in one of the two pedigrees showing a microdeletion (XLP-D and XLP$G$ ) or by the presence of genetic heterogeneity (two closely linked genes for X LP on the X chromosome). $W$ hile the latter hypothesis is based on several examples of $X$-linked genes, the former cannot be excluded until a candidate XLP gene is cloned and point mutations can be identified in all XLP patients.

\section{Acknowledgements}

We acknowledge M s M onica Scaranari (G aslini, G enova), M s L aetitia B outrand, M rs M arie-France Lavoué, M rs Suzanne Pauly, M s Tatiana Tocco, M s H élène D elage (IA R C, Lyon) for their technical assistance, and Dr Gyorgy Simon for supporting, maintaining data bases and developing the cgi-bin interface for R epM ask at TIG E M. We thank D r M ita M ancini (the YAC screening centre DIBIT, Milan) for providing the PAC library R PCl-5, Dr Tiziana Musso (Turin, Italy) for providing total RNA from human monocyte cell line. This work was supported in part by Ligue National Française contre le Cancer (LIGUE), Comité Départemental du Rhône. GP was funded by grants from the A ssociazione Italiana per La Ricerca sul Cancro (AIRC) and from the Italian TELETHON (E 294). The work carried out at the Sanger Centre was supported by the Wellcome Trust. J $S$ was supported by NIH grant IROIAI33532OIA 3. A B and FH were supported by fellowships from the LIGUE and $F H$ also by an ARC fellowship and a IA R C Special Training A ward.

\section{References}

1 Purtillo DT, Yang JP, Cassel CK et al: X-linked recessive progressive combined variable immunodeficiency (D uncan's disease). L ancet 1975; 1: 935-940.

2 Skare J C, M ilunsky A, Byron KS, Sullivan J L: Mapping the $\mathrm{X}$-linked lymphoproliferative syndrome. Proc $\mathrm{Nat}$ A cad Sci USA 1987; 84: 2015-2018.

3 Skare J C, G rierson H L, Sullivan J L et al: L inkage analysis of seven kindred with the $X$-linked lymphoproliferative syndrome (XLP) confirms that XLP locus is near DXS42 and DXS37. H um G enet 1989; 82: 354-358.

4 Sylla BS, Wang Q, Hayoz D, Lathrop GM, Lenoir GM: Multipoint linkage mapping of the $X q 25-26$ region in a family affected by the $X$-linked lymphoproliferative syndrome. Clin G enet 1989; 36: 459-462.

5 Wyandt $H E$, Grierson $H L$, Sanger WG, Skare JC, M ilunsky A, Purtilo DT: Chromosomal deletion of X q25 in an individual with $X$-linked lymphoproliferative disease. A m J M ed G enet 1989; 33: 426-430. 
6 Skare J, Wu B L, M adan S et al: Characterisation of three overlapping deletions causing $X$-linked lymphoproliferative disease. G enomics 1993; 16: 254-255.

$7 \mathrm{Wu} B \mathrm{~L}, \mathrm{Milunsky} \mathrm{A}, \mathrm{Nel}$ son $\mathrm{D}$ et al: High-resolution mapping of probes near the $X$-linked lymphoproliferative disease (XLP) locus. Genomics 1993; 17: 163-170.

8 Lamartine J, Nichols KE, Y in L et al: Physical map and cosmid contig encompassing a new interstitial deletion of the $X$-linked lymphoproliferative syndrome region. E ur J H um G enet 1996; 4: 342-351.

9 Lanyi A, Li BF, Li SB et al: A yeast artificial chromosome (YAC) contig encompassing the critical region of the $X$-linked lymphoproliferative disease (X LP) locus. G enomics 1997; 39: 55-65.

10 Purtilo D, Deflorio D, H utt $L$ et al: Variable phenolypic expression of an $\mathrm{X}$-linked recessive lymphoproliferative syndrome. N Engl J Med 1977; 297: 1077-1080.

11 Church D M, Stotler CJ, R utter J L, M urrell J R, Trofatter JA, B uckler A J : I solation of genes from complex sources of mammalian genomic DNA using exon amplification. $\mathrm{N}$ at $\mathrm{G}$ enet 1994; 6: 98-105.

12 Repetto $M$, Ballabio $A$, Zollo $M$ : A method to direct sequence cosmid Lawrist 16 clones. DNA Sequence 1997; 7: 229-234.

13 Ü berbacher EC, Mural RJ: Locating protein-coding regions in human DNA sequences by a multiple sensorneural network approach. Proc Natl A cad Sci USA 1991; 88: 11261-11265.
14 Solovyev VV, Salamov A A, Lawrence CB: Predicting internal exons by oligonucleotide composition and discriminant analysis of spliceable open reading frames. Nucleic A cids Res 1994; 22: 5156-5163.

15 A Itschul SF, Gish W, M iller W, M yers EW, Lipman DJ : Basic local alignment search tool. J Mol Biol 1990; 215: 408-410.

16 Durbin R, Mieg JT: The ACEDB Genome Database. Computational Methods in Genome Research 1994; Plenum 45-55.

17 Porta G, M acM illan S, Nagaraja R et al: 4.5-M b YAC STS contig at $50-\mathrm{K} b$ resolution, spanning $\mathrm{X} q 25$ deletions in two patients with lymphoproliferative syndrome. $G$ enome Res 1997 7: 27-36.

18 D ib C, Faure S, Fizames $C$ et al: A comprehensive genetic map of the human genome based on 5.264 microsatellites. N ature 1996; 380: 152-154.

19 Pinkel D, Straume T, G ray J W: Cytogenetic analysis using quantitative, high-sensitivity, fluorescence hybridization. Proc Natl A cad Sci USA 1986; 83: 2934-2938.

20 Y okoi H, H adano S, Kogi N, K ang X, Wakasa K, Ikeda J E : I solation of expressed sequences encoded by the human $\mathrm{Xq}$ terminal portion using microclone probes generated by laser microdissection. G enomics 1994; 20: 404-411.

21 Bedell MA, Jenkins NA, Copeland NG: Good genes in bad neighbourhoods. N at G enet 1996; 12: 229-232. 